\title{
NUCLEAR EMISSION MICROSCOPIES
}

\author{
B. L. Doyle ${ }^{\mathrm{a},{ }^{*}}$, D. S. Walsh" ${ }^{\mathrm{a}}$ S. N. Renfrow ${ }^{\mathrm{a}, \mathrm{b}}$, G. Vizkelethy ${ }^{\mathrm{a}, \mathrm{c}}$, \\ T. Schenkel ${ }^{d, e}$ and A.V. Hamza \\ a Sandia National Laboratories, Albuquerque, NM 87185, USA \\ $b$ Mission Research Corp. Huntsville, AL \\ $c$ on leave from Idaho State University, Pocatello, ID \\ $d$ Lawrence Berkeley National Laboratory, Berkeley, CA \\ e Lawrence Livermore National Laboratory, Livermore, CA
}

Alternatives to traditional nuclear microprobe analysis emerged two years ago with the invention of Ion-Electron Emission Microscopy (IEEM). With Nuclear Emission Microscopy the ion beam is only partially focused so as to fill the field of view of a special emission particle microscope system fitted with a single particle Position Sensitive Detector (PSD). When a single ion strikes the sample, the emitted secondaries (e.g. electrons, photons, ions, ...) are projected at great magnification onto this PSD where position signals are generated. These $\mathrm{X}$ and $\mathrm{Y}$ signals are then put into coincidence with the IBA signal(s) made by this same ion in a fashion completely analogous to traditional NMA. In this paper, an update will be given on the state of Nuclear Emission Microscopies, which currently includes IEEM and High Charged IonSecondary Ion Mass Spectroscopy (HCI-SIMS). In addition, a new type of full-field nuclear imaging is proposed: Ion-Photon Emission Microscopy or IPEM.

Sandia is a multiprogram laboratory operated by Sandia Corporation, a Lockheed Martin Company, for the United States Department of Energy under Contract

DE-AC04-94AL85000. 


\section{DISCLAIMER}

This report was prepared as an account of work sponsored by an agency of the United States Government. Neither the United States Government nor any agency thereof, nor any of their employees, make any warranty, express or implied, or assumes any legal liability or responsibility for the accuracy, completeness, or usefulness of any information, apparatus, product, or process disclosed, or represents that its use would not infringe privately owned rights. Reference herein to any specific commercial product, process, or service by trade name, trademark, manufacturer, or otherwise does not necessarily constitute or imply its endorsement, recommendation, or favoring by the United States Government or any agency thereof. The views and opinions of authors expressed herein do not necessarily state or reflect those of the United States Government or any agency thereof. 


\section{DISCLAIMER}

Portions of this document may be illegible in electronic image products. Images are produced from the best available original document. 


\section{Introduction}

A radically new form of nuclear microscopy, Nuclear Emission Microscopy (NEM), was invented by Sandia two years ago to address the problem of performing single-ion Nuclear Microprobe Analysis (NMA) using ions that are difficult, or even impossible, to focus with conventional nuclear microprobes [1]. The basic premise of NEM is that it is sometimes easier to determine where an unfocused ion hits a sample rather than focus and scan an ion to a prespecified location. The specific challenge Sandia was facing was the execution of Radiation Effects Microscopy $[2,3]$ to measure single event effects (SEE) on integrated circuits using high Linear Energy Transfer (LET) beams from the Sandia Tandem-Radio Frequency Quadrupole (RFQ) accelerator. Figure 1 compares the surface LET, depth of penetration (both in $\mathrm{Si}$ ) and mass-energy products of beams from several US accelerators used for SEE testing. The highest LET ions of most accelerators, including the Sandia Tandem-RFQ, used for SEE measurements, have very high magnetic rigidity and poor energy chromaticity which makes microfocusing quite problematic. In addition some of these ions can easily penetrate the tips of the object and aperture slits used to define these beams, which further deteriorates the spatial definition of such beams.

The first NEM was called Ion Electron Emission Microscopy or IEEM [1]. With IEEM, the ion beam is not focused, but instead, the secondary electrons made by each ion on the target are project imaged through a set of electrostatic lenses onto a single electron position sensitive detector (PSD). The signals from this PSD then records, with resolution potentially much higher than current microfocused $\mathrm{MeV}$ ion spots, the $\mathrm{X}, \mathrm{Y}$ strike point of the ion on the target, thereby ameliorating the need to focus the incident ion beam. After producing secondary electrons on the surface of the sample, the ion continues to penetrate into the semiconductor or device under test where it deposits charge, and potentially produces a single event effect [4].

Coincidentally, a second NEM was developed by LLNL almost simultaneously with IEEM which is called Highly Charged Ion Secondary Ion Mass Spectroscopy (HCISIMS) [5]. HCI-SIMS is actually quite similar to IEEM in that secondary electrons are also project imaged to provide the ion strike point, but in this case the incident ions are lower-energy highly charged ions from an Electron Beam Ion Trap (EBIT), and the detected signal is the measurement of the time of flight of secondary ions produced during the interaction of these ions with the sample surface. HCI-SIMS is therefore an NEM that images surface composition.

In this paper, an update is given on the status of the two existing types of Nuclear Emission Microscopy: IEEM and HCI-SIMS. A new full field nuclear microscopy being developed at Sandia, Ion Photon Emission Microscopy (IPEM) is also proposed. IPEM will be virtually identical to IEEM, with the exception that single-ion-induced photons are projection imaged, instead of electrons. Some of the first proof-of-concept experiments for IPEM will be discussed, as will the potential future utility of IPEM. One of the most exciting predictions regarding IPEM is that it could be performed with radioactive sources in air, utilizing a standard optical microscope.

RECEIVED 


\section{Nuclear Emission Microscopies}

Single-ion nuclear microscopy normally involves focusing a low intensity (a few fA) $\mathrm{MeV}$-energy ion beam to micron or even submicron dimensions, steering this beam to a specific $(X, Y)$ position on the sample specimen, and measuring an analysis signal such as IBICC (Note that we could as well be discussing SEU-Imaging, time resolved (TR)IBICC, Ion Microbeam Tomography, the detection of sputtered ions (SIMS), or any IBA involving single incident ions.) produced when an individual ion enters into the sample. These analysis signals are typically recorded as a pulse height (S) at that position. The process is then repeated at a new $(\mathrm{X}, \mathrm{Y})$ position, which is reached by scanning the beam in a type of "flying spot" analysis. A schematic of this form of nuclear microprobe analysis (NMA) is shown in Fig. 2a. The data from such an experiment is usually collected in list-mode, i.e. a file of $\{\mathrm{X}, \mathrm{Y}, \mathrm{S}\}$ events, which is later binned into a Counts $(\mathrm{X}, \mathrm{Y}, \mathrm{S})$ matrix of data for analysis and the creation of data images.

As indicated above, an alternative to this traditional style of nuclear microprobe analysis emerged two years ago with the introduction of Nuclear Emission Microscopies, the first being IEEM. With this new type of microscopy (shown in Fig. 2b), the ion beam is either unfocused or only partially focused so as to fill the field of view of a special lens system that witnesses the ion-solid interaction that occurs at or near the sample surface. When a single ion strikes the sample, the emitted particles (electrons, photons, ions, ...) are projected at great magnification onto a highly sensitive Position Sensitive Detector (PSD) which generates $X_{p}$ and $Y_{p}$ signals. In some cases, a thin coating optimized to produce large numbers of these particles is applied to the sample surface. These PSD signals are then put into coincidence with the analysis signal (S) made by this same ion, inside the sample, and an $\left\{X_{p}, Y_{p}, S\right\}$ event file is created. This file is virtually identical to the $\{\mathrm{X}, \mathrm{Y}, \mathrm{S}\}$ list made by traditional nuclear microscopy, where the only difference is knowing the magnification power factor of the particle lens projection system.

Nuclear Emission Microscopy is distinguished from other full field microscopies [6], such as Photoelectron Emission Microscopy (PEEM), Low Energy Electron Microscopy (LEEM), or Stigmatic Imaging Secondary Ion Mass Spectrometry (SIMS), because it relies on the combination of two completely unrelated signals: the emission particles from the coating layer which is used only to determine $(X, Y)$ and the measurement event signal created inside the sample (e.g. IBICC). With other full field microscopies, the emitted particles carry not only the $(X, Y)$ position information, but also the information being measured by the analysis (e.g. work function variations with PEEM, surface steps with LEEM, and composition with SIMS). HCI-SIMS fits somewhere in the middle of these two types of emission microscopies because the emitted particles (electrons and ions) used for position also provide the start pulses used in the time-of-flight analysis.

One of the great attributes of the traditional Nuclear Microprobe Analysis (NMA) analysis is that each and every ion can produce an analysis signal (e.g. IBICC). This unity detected event probability guarantees that this type of analysis minimizes the amount of radiation damage to the sample. This is not necessarily the case with the Nuclear Emission Microscopy method, as has been discussed previously [1]. To 
illustrate this, refer to Fig. $2 \mathrm{~b}$. When the individual ion strikes the sample lets say that $\gamma$ emitted particles are produced. The successful detection of these particles by the PSD depends on the collection and transmission efficiency, $\varepsilon$, of the projection microscope lens system together with the detection efficiency, $\delta$, of the PSD. So, of the N particles produced by the ion on the sample, only $\gamma \varepsilon \delta$ are detected. Several limiting cases, numbered (1-4), then present themselves.

(1) If $\gamma \varepsilon \delta<<1$, count rates could become so low that the associated long analysis times or huge damage levels would preclude this application.

(2) If $\gamma \varepsilon \delta<1$, then NEM will have a lower count rate than NMA for the same beam current. This means that for the same data quality (i.e. statistics), the sample will suffer from more radiation damage with NEM. This may or may not represent a problem.

(3) If $\gamma \varepsilon \delta>1$, then the quality of data from the NEM and NMA analysis and the degree of radiation damage should be comparable. For this case multiple particles will be detected for each ion, but they all originate from the same point and therefore do not confuse the PSD.

(4) If $\gamma \varepsilon \delta>>1$, then so many emission particles can potentially be detected, that closing the apertures in the microscope (to improve resolution) will have no cost in count rate, that is until $\gamma \varepsilon \delta=1$. It is in this situation that NEM has the potential of providing superior resolution as compared with NMA.

\section{Examples}

There are currently three Nuclear Emission Microscopies at various stages of development: Ion-Electron Emission Microscopy (IEEM), Ion-Photon Emission Microscopy (IPEM), and Highly Charged Ion - Secondary Ion Mass Spectroscopy (HCISIMS). Table 1. summarizes attributes of each of these NEMs.

Table 1. Properties of Nuclear Emission Microscopies.

\begin{tabular}{|c|c|c|c|c|c|c|c|c|c|c|}
\hline NEM & beam & energy & $\begin{array}{c}\text { emitted } \\
\text { ptcl }\end{array}$ & $\gamma$ & $\varepsilon$ & $\delta$ & $\begin{array}{l}\text { det. } \\
\text { ptcll } \\
\text { ion }\end{array}$ & $\begin{array}{l}\text { detected } \\
\text { signal }\end{array}$ & $\begin{array}{l}\text { property } \\
\text { measured }\end{array}$ & $\begin{array}{l}\text { resolution } \\
\text { (um) }\end{array}$ \\
\hline IEEM & $\mathrm{He}$ & $6 \mathrm{MeV}$ & e- & 4 & 0.1 & 0.5 & 0.2 & $I B I C C, \ldots$ & elec. properties & 1 \\
\hline & $\mathrm{Au}$ & $380 \mathrm{MeV}$ & & 360 & 0.1 & 0.5 & 18 & IBIC & elec. properties & 0.1 \\
\hline IPEM & $\mathrm{He}$ & $6 \mathrm{MeV}$ & photon & 2000 & .0002 & 0.25 & 0.1 & $|B| C C, \ldots$ & elec. properties & 1 \\
\hline HCl-SIMS & $X e^{48+}$ & $0.7 \mathrm{MeV}$ & e- & 100 & 0.1 & 0.5 & 5 & sputtered - ion & surface compo & 0.8 \\
\hline $\mathrm{HCl}$-SIMS & $X e^{48+}$ & $0.7 \mathrm{MeV}$ & $\mathrm{H}+$ & 10 & 0.1 & 0.5 & 0.5 & sputtered + ion & surface compo & 2 \\
\hline
\end{tabular}




\subsection{Ion-Electron Emission Microscopy (IEEM)}

Referring to Fig. $2 \mathrm{~b}$ and reference [1], with IEEM a beam of $\mathrm{MeV}$ ions, provided by an accelerator, impinges on a semiconductor sample (currently at an angle of $75^{\circ}$ with the surface normal) which is usually coated with a thin metal layer to enhance production of particles, which in this case are secondary electrons. The secondary electrons which are generated by each ion (and shown to originate at X,Y in Fig. 2) are then accelerated and projected using the lens system of a Photon Electron Emission Microscope (PEEM). The incident "flood" ion beam is usually partially focused with a magnetic quadrupole lens, so as to fill the field of view of the PEEM. These electrons are refocused to positions $X_{p}$ and $Y_{p}$ at the projection focal plane of the PEEM and recorded with a microchannel plate + resistive anode encoder Position Sensitive Detector (PSD). Two electronic pulses with voltages proportional to $X_{p}, Y_{p}$ are generated by the $P S D$, and these signals are then put into coincidence with the analysis signal's pulse height $\mathrm{S}$ (shown schematically as IBICC in Fig. 2) using a multiparameter data acquisition system. Each event is stored as an $\left\{\mathrm{X}_{\mathrm{p}}, \mathrm{Y}_{\mathrm{p}}, \mathrm{S}\right\}$ vector element in list mode.

Hasselkamp et al. [7] have shown that the number of secondary electrons $\left(\gamma_{\mathrm{e}}\right)$ produced by each ion can be expressed as:

$$
\gamma_{\mathrm{e}}=\Lambda(\mathrm{dE} / \mathrm{dx})
$$

for ions on metals at normal incidence. $\Lambda$ ranges from 0.07 to 0.13 for various ions on smooth silver targets when the stopping power is expressed in units of eV/A. The values of $\gamma$ listed in Table 1 for IEEM using beams of $6 \mathrm{MeV} \mathrm{He}$ and $380 \mathrm{MeV} \mathrm{Au}$ were obtained using scaled stopping powers [8] for these ions on $\mathrm{Ag}$ and assuming $\Lambda=0.1$. In [1] we measured the total system detection efficiency to be 0.05 using protons on $\mathrm{Au}$ coated PIN diodes using the largest aperture in the PEEM (300 um). This indicates that for a PSD efficiency of $\delta=0.5$, that $\varepsilon=0.1$, and these values are also listed in the table. The detected particles per ion, which in this case are electrons, will range from $\sim 0.2$ to almost 20 for IEEM using ions at normal incidence. For a $75^{\circ}$ tilt, $\gamma_{\mathrm{e}}$ increases by a factor of $\sim 4$ according to the Hasselkamp theory. The resolution of IEEM was measured to be 0.9 um using the 300 um aperture in the PEEM lens.

This analysis suggests that for the largest PEEM aperture, that using He ions at normal incidence will fall under case (2) above where micron-level resolution can be obtained, but with $\sim 5 \mathrm{x}$ more beam-induced damage to the sample. Using He ions incident at $75^{\circ}$ will fall into case (3) where IEEM and NMA are essentially equivalent. The use of $\mathrm{Au}$ ions falls into case (4), where multiple electrons are detectable, and this indicates that smaller PEEM apertures could be used to improve the 0.9 micron resolution. If a 70 um aperture is used, the detected electrons/ion should drop to 1 and the resolution should improve to $\sim 0.2 \mathrm{um}$.

A good example of IEEM is shown in Fig. 3. A 1 fA, $5 \mathrm{MeV}$ He beam at $75^{\circ}$ incidence was used to produce these images for a prototype Au-coated Cadmium Zinc Telluride 
(CZT) gamma-ray detector. Fig. 3 a shows the secondary electron intensity map. The partial focus of the beam can easily be discerned as the bright parallelogram near the center region of this image. Note that there are counts at the 1-2 electrons per pixel level outside this region due to the incomplete focus of the beam or halo effects. In Fig. 3b we plot the IBICC map collected using the $(X, Y)$ position information of the secondary electrons in $4 \mathrm{a}$ in conjunction with the IBICC pulses measured from this sample. The median of the IBICC pulse height distribution at each $(X, Y)$ position pixel is being plotted in this figure. It is interesting to note that while the quality of the IBICC image is best in the region of the partial focus, structure can even be observed outside this area where the beam intensity was extremely low. While this particular sample did not demonstrate significant beam damage effects, the fact that an image can be seen in the halo region surrounding the beam spot indicates that considerably less beam exposure could have been used to obtain this data. While it is not currently clear whether the variations in this IBICC signal are due to defects in the Au layer or the CZT crystal, it is apparent from this figure that IEEM-IBICC can provide data of nearly the same quality as a scanned nuclear microprobe.

Unfortunately, this was not found to be the case when examining ICs with IEEM-IBICC images taken with $5 \mathrm{MeV}$ He on a Sandia TA-780 16K SRAMs. While individual transistors were clearly defined using traditional microbeam IBICC, they could not be resolved with the IEEM-IBICC. This was probably due to the fact that the He ion beam used in this experiment impinged at an angle of $75^{\circ}$ to the surface normal. Since the emitted electrons come off at normal incidence, this IBICC image suffers from a type of parallax where the position of the IBICC event does not correspond to the generation point of the corresponding electrons.

\subsection{Ion-Photon Emission Microscopy - IPEM}

A natural extension of IEEM is to use ion-induced photons (ionolumenescence) to record the arrival point of ions onto a sample. This technique is called Ion Photon Emission Microscopy or IPEM. Anyone who has used a quartz to view an ion beam of an accelerator has performed a crude form of IPEM where, referring to Fig. 2, the coating on the sample is the quartz, the emission particles are photons, the lens is in your eye, the PSD is your retina, and the analyzer is your brain! To our knowledge, no one has actually performed an IPEM experiment where an ion induced signal in the target is correlated with the position where photons are created. This is therefore the first time such a system has been proposed. We present here, proof of principle experiments where $\gamma, \varepsilon$, and $\delta$ are determined for IPEM, and then predict the performance characteristics of a system which is currently under development at Sandia.

Refer to Fig. 2b and Fig. 4 for the following discussion. With IPEM the beam of MeV ions (from an accelerator or radioactive source) impinges on a sample which is coated with a thin phosphor layer to enhance production of photons. The photons generated by each ion are then collected and projected using the lens system of an optical microscope (OM). We are currently using the JOEL OM-40 in-vacuo optical microscope that is also used for front-viewing applications on our conventional nuclear microprobe. This 
particular microscope is not shown in the figure (a conceptual microscope is shown in Fig. 4), but it has a $1 \mathrm{~mm}$ hole in a prism followed by the objective lens through which the beam passes to the target at normal incidence. The photons generated by the beam are 1) collected and made parallel by this same lens, 2) passed to the prism where they are bent $90^{\circ}$ and away from the beamline, 3) passed to another prism and another $90^{\circ}$ bend, and 4) on to the eyepiece lens which focuses the photons onto the single photon PSD. In the future, this single photon PSD will consist of a bialkali photon-electron converter + microchannel plate + resistive anode encoder, but currently we are just using a Hamamatsu miniaturized photo multiplier tube (PMT). This PMT can detect single photons, but cannot resolve their position. The rest of the data acquisition follows that of the IEEM [1] which correlates an analysis signal(s) with the X,Y signals from the PSD, as shown in Fig. 4.

A companion paper [9] gives details of our quest to find the optimum phosphor coating for IPEM. In general, we have observed that for standard phosphors the number of photons detected per incident ion ranges from $\sim 0.2$ to $\sim 10$ for thick phosphors, and $\sim 0.05$ for thin phosphor blades.

The Birks ionoluminescence [10] theory gives:

$$
\gamma_{\mathrm{p}}=(\mathrm{dL} / \mathrm{dx}) \mathrm{X}
$$

where

$$
\frac{d L}{d x}=\frac{P \cdot\left(\frac{d E}{d x}\right)}{1+\beta \cdot\left(\frac{d E}{d x}\right)}
$$

and $\mathrm{P}$ is the number of photons produced per $\mathrm{MeV}$ (16666 for anthracene), $\mathrm{dE} / \mathrm{dx}$ is in units of $\mathrm{MeV} /$ micron, $\beta$ represents a saturation in luminescence and is in units of microns $/ \mathrm{MeV}$ (117 for anthracene), and $\mathrm{X}$ is the thickness of the phosphor in microns. Using scaled stopping powers [8] and an anthracene phosphor layer 20 microns thick (anthracene is an organic crystal to which other phosphors are often compared), the number of photons made by each $6 \mathrm{MeV}$ He ion, $\gamma_{\mathrm{p}}$ is 2000 . Because eq. 3 is in already in saturation for $\mathrm{He}, \mathrm{Au}$ ions only produce a modest increase in photon generation. The collection-transmission efficiency of the OM-40 is $\varepsilon \sim 0.0002$, and the efficiency of the PSD will be in the $\delta \sim 0.2$ range. The number of photons/ion will then be $\sim 0.1$ for an average 20 micron phosphor, for either $\mathrm{He}$ or $\mathrm{Au}$ ions. This places IPEM in case (2) above, for both the He and $\mathrm{Au}$ ions, where increased ion damage is an issue. We expect IPEM to move to case (3) or even (4) when optimum phosphor coating layers are used.

A proof of principle IPEM test was made using the Sandia nuclear microprobe and the OM-40 microscope fitted with a PMT. A $20 \mathrm{MeV} \mathrm{C}$ beam was focussed and then scanned across a sample consisting of a PIN diode coated with 5-10 microns of a very 
nonuniform $\mathrm{SrGa}_{2} \mathrm{~S}_{4}: \mathrm{Eu}$ thiogallate phosphor (used for green in commercial projection TVs) deposited by sedimentation. The position of the ions striking the sample was controlled by the Sandia microbeam focussing and steering system, and not by detecting the position of the photons using a PSD. In fact, IPEM would never work very well with such a ceramic polycrystalline phosphor because the lateral resolution would be determined by the crystallite size and other light scattering effects, rather than the microscope+PSD. Nevertheless, IL and IBICC images were made by combining the pulse height signals generated by both the PMT and PIN diode with the X,Y scan position, and these images are plotted in Fig. 5a and 5b. Fig. 5a plots the median of the PMT output vs. X,Y which approximately corresponds to the number of photons per ion vs. X,Y. The phosphorescence of thiogallate has a lifetime of only $\sim .2$ us when excited with $\mathrm{C}$ ions, and therefore most of the photons generated, by each ion, are collected as a single pulse in the PMT and associated electronics, in a fashion similar to pile-up. The blue region represents single photon detection presumably from thin regions while the yellow areas indicate as many as 3 to 4 photons are being collected, all at once, from thicker regions. Thallogallate is known to be much brighter than anthracene, as demonstrated by this data. Fig. $5 b$ shows a medium filtered IBICC image made simultaneously with 5a. Here the contrast is reversed: the yellow regions have the highest IBICC medians, and therefore are regions of the thinnest phosphors; whereas, the blue areas have the lowest energy IBICC signals, and demonstrate the greatest energy loss from passage through thick phosphor regions. The black areas in both images show a lack of coincidences, which means that no phosphor was present in these areas. While this experiment is not exactly IPEM, it does demonstrate the viability of this new emission microscopy.

It is clear that superior phosphors will be required to fully realize the potential of IPEM. These phosphors should have the following attributes:

1. be thin ( $<5 \mathrm{um})$ (so that ions can easily penetrate into the sample)

2. be easy to apply (to facilitate sample preparation)

3. have high brightness (i.e. photons/ion/um)

4. be clear and continuous (to avoid photon-blooming due to scattering from defects)

5. have a low index of refraction (to increase the collection angle)

6. emit in the red (most PSDs have highest efficiency for red)

7. possess an attenuation coefficient comparable to the film thickness (to reduce multiple scattering induced broadening).

Much work is being undertaken to find the optimum phosphor for IPEM [9]. Once this phosphor is determined, the OM-40 at Sandia will be fitted with a single photon PSD and true IPEM will be performed.

\subsection{Highly Charged Ion Secondary Ion Mass Spectoscopy (HCI-SIMS)}

Highly charged ions ( $\mathrm{HCI}$ ), like $\mathrm{Xe}^{48+}$ or $\mathrm{Au}^{69+}$, with velocities below the Bohr velocity, or kinetic energies $\leq 5 \mathrm{keV} / \mathrm{u}$, have charge states far in excess of the mean equilibrium charge state that corresponds to the velocity with which they impinge on a solid target. 
The latter is about $1+$. Measurements with thin foil targets have shown that charge state equilibrium is established within a few fs $[11,12]$. During this time, the potential energy associated with the initial charge state is deposited close to the target surface. In the example of $\mathrm{Au}^{69+}$ with a kinetic energy of a few hundred $\mathrm{keV}$, a potential energy of 170 $\mathrm{keV}$ is deposited along a path of about $50 \mathrm{~A}$ in amorphous carbon. The mean energy deposition rate of about $3.4 \mathrm{keV} / \mathrm{A}$ is close to the SRIM value of the maximum electronic stopping power for $600 \mathrm{MeV}$ gold ions in carbon $(2.6 \mathrm{keV} / \mathrm{A})$. The intense, localized and ultrafast electronic excitation of a surface by the equilibration of a slow, highly charged ion results in high yields of secondary electrons, positive and negative secondary ions as well as molecular ions. These high yields are used to image surfaces in the highly charged ion based emission microscope that was developed at Lawrence Livermore National Laboratory: HCI-SIMS [11,5].

Again referring to Fig. $2 b$, with HCI-SIMS the incident highly charged ions are produced in an Electron Beam Ion Trap or EBIT, and accelerated as a flood beam to the sample. An objective (immersion) lens accelerates the particles emitted at the surface, which in this case are either secondary electrons (like IEEM) or $\mathrm{H}+$ ions ( $\mathrm{H}$ is ubiquitous on sample surfaces) through the flight tube to a position sensitive micro channelplate detector (PSD). The flight time between start pulses from electrons (in negative polarity) or protons (in positive polarity) and stop pulses from trailing secondary ions is used to determine the mass-to-charge ratio of the secondary ions. Secondary electron yields of a few hundred have been observed using HCI-SIMS [11], which is consistent with the Hasselkamp theory (Eq. 1) above and the $3400 \mathrm{eV} / \mathrm{A}$ stopping powers. For positivemode HCI-SIMS, the $\gamma$ for protons is $\sim 10$. These values and lens and PSD performance are listed in Table 1. It can be seen in this table that HCI-SIMS for negative ion analysis falls into case 4, whereas for case (3) applies for positive ions. Using slow, highly charged ions as projectiles also has the potential to increase sensitivity due to increased ionization probabilities of secondary ions [13].

The first prototype instrument has demonstrated a resolution of $800 \mathrm{~nm}$ in the imaging of copper lines on silicon based on secondary electron contrast [5]. In positive polarity, a resolution of $2 \mu \mathrm{m}$ was achieved. The investigation of resolution limiting factors is in progress.

The fact that multiple secondary ions are emitted for individual projectiles allows the application of coincidence counting to HCI-SIMS $[14,15,16]$. Here, time-of-flight cycles are started by the impact of an individual $\mathrm{HCI}$ and streams of secondary ions associated with this start pulse are stored in a list. After accumulation of a few million cycles, the list can be searched for TOF-cycles in which a specific secondary ion was detected. Summation of all these cycles now shows other secondary ions that were emitted from individual projectiles together with the specified secondary ion. Through this analysis of secondary ion coincidences, information is gathered on the nano-environment of a selected species. The length scale of the composition information is given by the area from which one $\mathrm{HCI}$ emits secondary ions. We estimate the latter to be 10 to $20 \mathrm{~nm}$ in diameter. This effective resolution is similar to the practical limit in focused ion beam 
SIMS [17]. HCI-SIMS with coincidence analysis has been applied to the characterization of processing steps in semiconductor manufacturing.

In Fig. 6, we show an example of a relatively low resolution image of a scratch on a quartz lens with $700 \mathrm{keV} \mathrm{Xe} \mathrm{e}^{4+}$ ions. Contrast is based on secondary electron emission. Charge compensation was achieved with a pulsed low energy electron gun. Spectra of negative secondary ions collected in parallel with the electron pulse height image are shown in Fig. 7. The top spectrum shows secondary ions emitted from the scratched region. The spectrum from the unscratched region exhibits large peaks of surface contaminants. An important and challenging problem that is being addressed with $\mathrm{HCI}$ emission microscopy is that of damage mechanisms in optical components exposed to high power lasers. Here, the integration of coincidence analysis with emission microscopy at improved resolution holds the promise to enable significant advances.

\section{Conclusion and Future Directions}

It is clear from the above that Nuclear Emission Microscopy will play an increasingly important part in the future evolution of Nuclear Microscopy. IEEM is already starting to be used for Radiation Effects Microscopy of ICs and semiconductors. IPEM should provide an extremely simple platform to microscopically study the electronic properties of semiconductors. HCI-SIMS is proving to be a highly sensitive new surface analysis where, by using the mass-coincidence technique, particulates of only a few $\mathrm{nm}$ in size can be studied.

While the underlying motivation to develop these NEMs was to avoid focusing the beam, an additional advantage is now becoming apparent: Radioactive alpha or fission sources could potentially be combined with NEM to even eliminate the need for an accelerator. For example, an alpha-source IEEM would be quite compact, and trivial to implement in a laboratory associated with an IC manufacturing line, semiconductor R\&D facility, or any laboratory which does not possess an accelerator, but is interested in performing single-ion nuclear microscopy. One of the most exciting predictions regarding IPEM is that it could be performed in air with an alpha particle source coated onto the objective lens of an optical microscope (shown in Fig. 4), and replacing the CCD with a single photon PSD.

It is important to point out here that NEMs are not being proposed as a replacement to traditional nuclear microprobes, but that they do offer an attractive alternative in certain very specialized applications. Because of the low count rate capability of virtually all PSDs ( $<1 \mathrm{Mcps})$, these applications must be made with beam currents $<100 \mathrm{fA}$. Very few routine IBA techniques can be performed with such low beam currents. Until the bandwidth of PSDs increases dramatically, NEMs will therefore be limited to techniques which generally fall under the heading of single-ion nuclear microscopy, such as the various Radiation Effects Microscopies, Ion Transmission Microscopies and SIMS, all which have a near unity detected event probability. 
Future developments of these NEMs which are expected to occur in the next few years include:

IEEM- The only way to resolve the parallax problem is to use ions that impinge on the sample at normal incidence, and we are in the process of rebuilding the IEEM to do exactly that. This second generation IEEM will transmit the $\mathrm{MeV}$ ion beam directly through an annular PSD, through the PEEM lens system and onto the sample. Specially designed ion collimators have been positioned within the PSD to prevent beam ions from triggering the PSD. The low electric fields used to focus the secondary electrons are expected not to affect the trajectory of the $\mathrm{MeV}$ ions. The system is also being reassembled on the RFQ booster system.

IPEM- A Photec single photon PSD is being acquired which has 70 micron resolution. This PSD will replace the PMT on the OM-40 and should provide a final resolution in the 0.5 micron range. This first IPEM system will then be tested on samples which have been coated with the optimum phosphor layer determined by the Sandia-UNT-Legnaro collaboration [9]. After this test, we plan to coat an alpha particle source onto the objective lens of a high power optical microscope, and replace the CCD with the Photec single photon PSD. Such a system should be able to perform IPEM for radiation microscopy applications in air, with an instrument of low cost $(<\$ 60 \mathrm{~K})$ and tabletop footprint.

HCI-SIMS- Two groups at LLNL and LBNL are pursuing the continued development of this nuclear emission microscopy. Initial applications are expected to be found in the IC industry, for example, to measure the composition of nm-sized particulates which can reduce production yields. These groups are also exploring a compact alternative to a Tandem-RFQ booster system for the generation of high LET beams with energies around $1 \mathrm{MeV} / \mathrm{u}$ which involves the integration of an EBIT as a source for highly charged ions $\left(\mathrm{Fe}^{26+}, \mathrm{Xe}^{52+}\right.$ to $\mathrm{Au}^{77+}$ ) into the terminal of a 2 to $3 \mathrm{MV} \times \mathrm{q}$ accelerator structure.

\section{Acknowledgements:}

Work at Sandia supported by the U.S. DoE under contract DE-AC04-94AL85000. Work at LBNL was supported by the Director, Office of Basic Energy Sciences, of the U. S. Department of Energy under Contract No. DE-AC03-76SF00098. 


\section{References:}

[1] Doyle BL, Vizkelethy G, Walsh DS, Senftinger B, Mellon M

NUCLEAR INSTRUMENTS \& METHODS IN PHYSICS RESEARCH

SECTION B-BEAM INTERACTIONS WITH MATERIALS AND

ATOMS , v. 158(\#1-4) pp. 6-17 SEP 1999

[2] HORN KM, DOYLE BL, WALSH DS, SEXTON FW

SCANNING MICROSCOPY, v. 5(\#4) pp. 969-976 DEC 1991

[3] Breese MBH

MATERIALS SCIENCE AND ENGINEERING B-SOLID STATE

MATERIALS FOR ADVANCED TECHNOLOGY, v. 42(\#1-3) pp. 67-76 DEC 15, 1996

[4] For an excellent review see "Single Event Phenomena", Messenger GC and Ash MS, Chapman and Hall (1997)

[5] Highly charged ion based time-of-flight emission microscope

Schneider DH

Hamza AV, Barnes AV, Magee E, Newman M, Schenkel T, McDonald JW,

REVIEW OF SCIENTIFIC INSTRUMENTS , v. 71(\#5) pp. 2077-2081

MAY 2000

[6] O.H. Griffith and W. Engel., Ultramicroscopy 36, 1 (1991)

[7] D. Hasselkamp, K.G. Lang, A. Scharmann, and N. Stiller, Nucl. Instrum. And Methods 180 (1981) 349.

[8] J. Zigeler, Appl. Phys. Lett. 31 (1977) 544

[9] Yang et. al (these proceedings)

[10] J.B. Birks, The Theory and Practice of Scintillation Counting, Pergamon Press, Oxford (1964)

[11] T. Schenkel, A. V. Hamza, A. V. Barnes, and D. H. Schneider, Prog. Surf. Science 61, 23 (1999)

[12] M. Hattass, T. Schenkel, A. V. Hamza, A. V. Barnes, M. W. Newman, J. W.

McDonald, T. R. Niedermayr, G. A. Machicoane, and D. H. Schneider, Phys. Rev. Lett. 82,4795 (1999) 
[13] T. Schenkel, A. V. Barnes, A. V. Hamza, J. C. Banks, B. L. Doyle, D. H. Schneider, Phys. Rev. Lett. 80, 4325 (1998)

[14] A. V. Hamza, T. Schenkel, A. V. Barnes, D. H. Schneider, J. Vac. Sci. Technol. A $17,303(1999)$

[15] T. Schenkel, M. W. Newman, T. R. Niedermayr, G. A. Machicoane, J. W.

McDonald, A. V. Barnes, A. V. Hamza, J. C. Banks, B. L. Doyle, K. J. Wu, Nucl. Instr. and Methods in Phys. Research B 161-163, 65 (2000)

[16] T. Schenkel, K. J. Wu, H. Li, M. W. Newman, A. V. Barnes, and A. V. Hamza, J. Vac. Sci. Technol. B 17, 2331 (1999)

[17] F. A. Stevie, et al., J. Vac. Sci. Technol. B 17, 2476 (1999) 


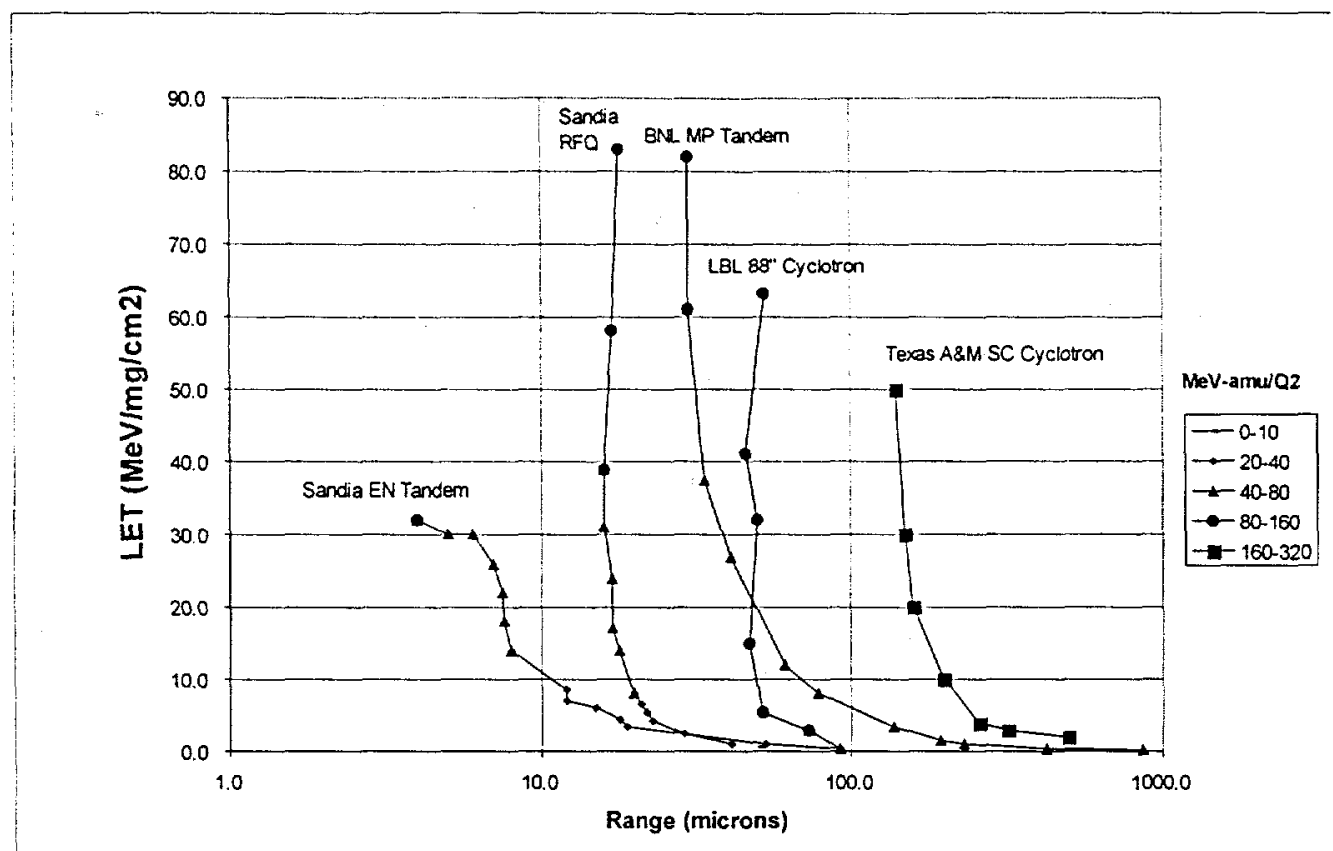

Fig. 1: Linear Energy Transfer (LET) vs. ion range and mass-energy product for beams from several US accelerator labs used for radiation effects testing of integrated circuits.

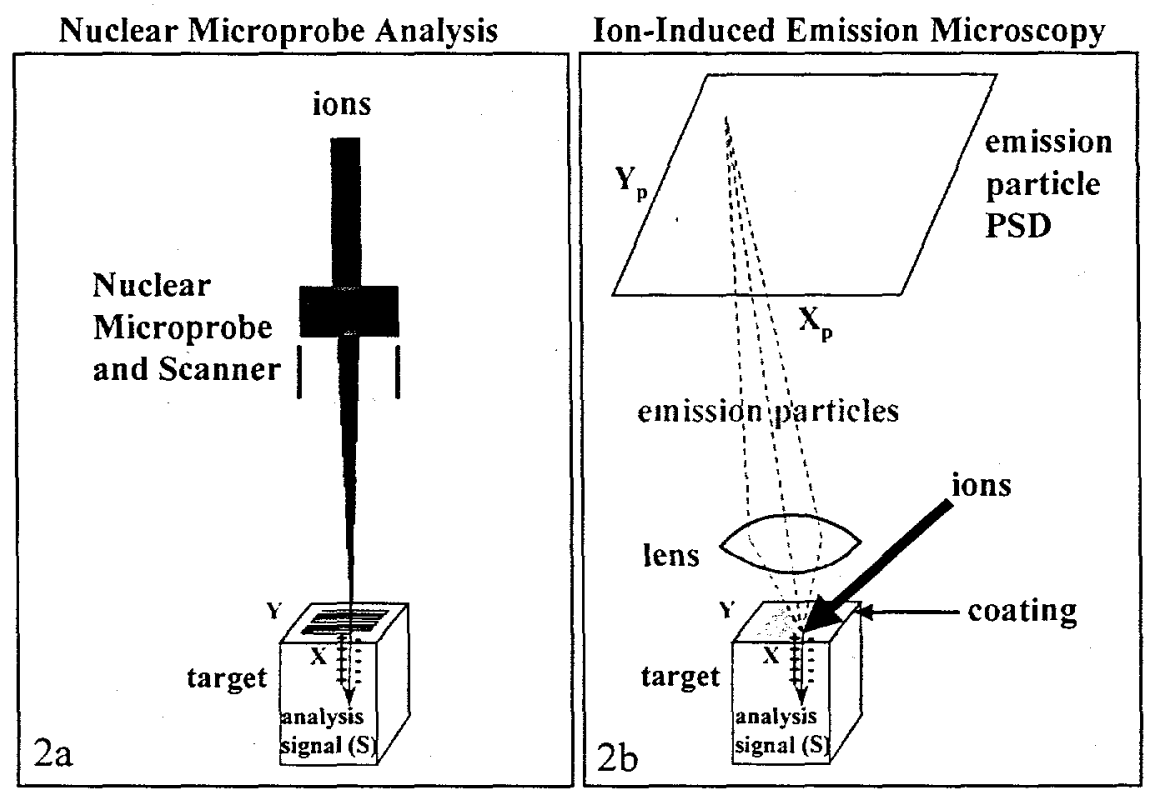

Fig. 2: Schematic comparison of traditional "Flying Spot" Nuclear Microprobe Analysis where the ion beam is focused and scanned, and lon-Induced Emission Microscopy where the beam is not focused, but instead emission particles produced by each ion are project imaged. 


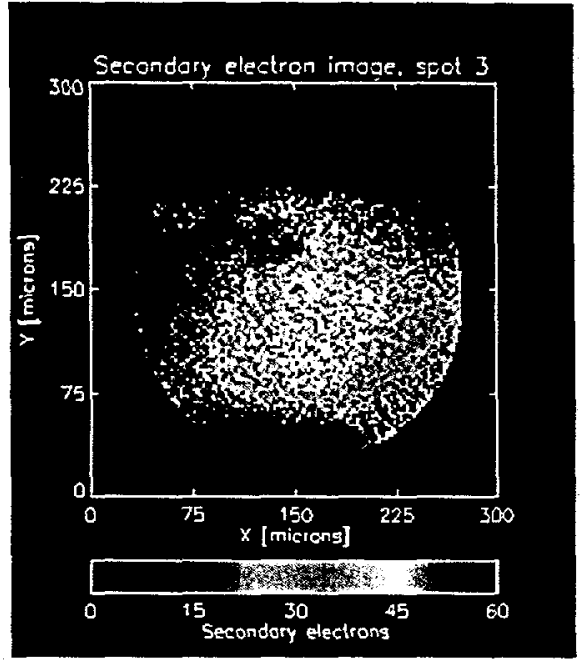

$3 a$

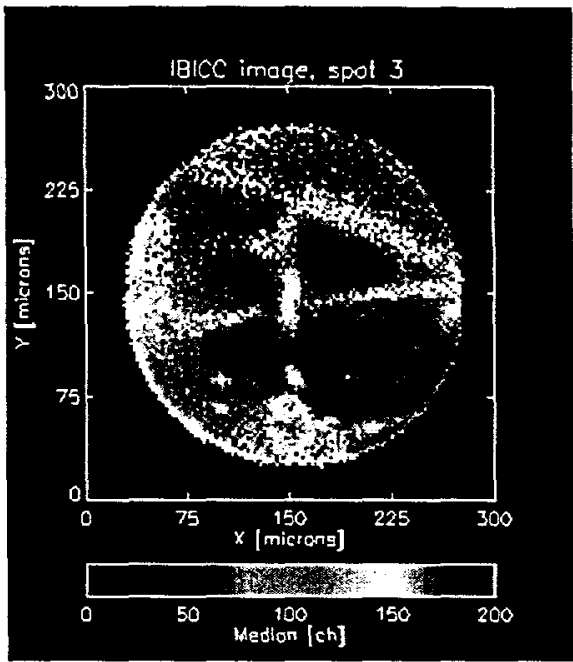

$3 b$

Fig. 3: Ion Electron Emission Microscopy (IEEM) images of (a) $20 \mathrm{MeV} \mathrm{C}$ secondary electron intensity and (b) Ion Beam Induced Charge Collection (IBICC) medians for a $\mathrm{CdZnTe}$ detector. While the beam was partially focused into the parallelogram shown in (a), the IBICC image in (b) is completely filled.

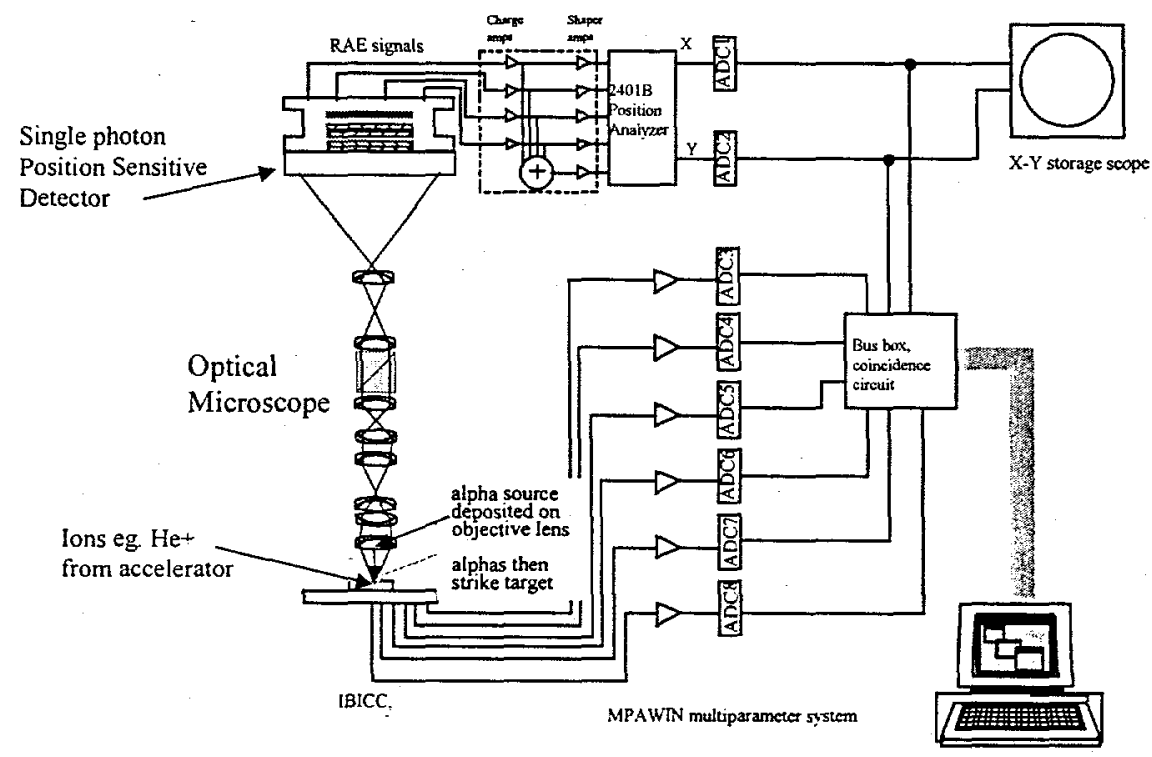

Fig. 4: Schematic diagram of an lon-Photon Emission Microscope (IPEM) system. Ions from either an accelerator or radioactive alpha source impinge on the sample producing an IBICC pulse, and simultaneously generate photons at the sample surface which are projected at high magnification onto a single photon position sensitive detector. A multiparameter system reassembles the position and IBICC signals into a list of data for analysis. 


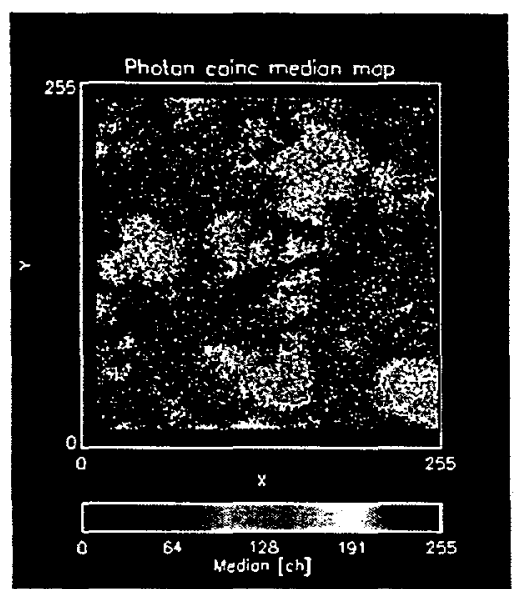

$5 \mathrm{a}$

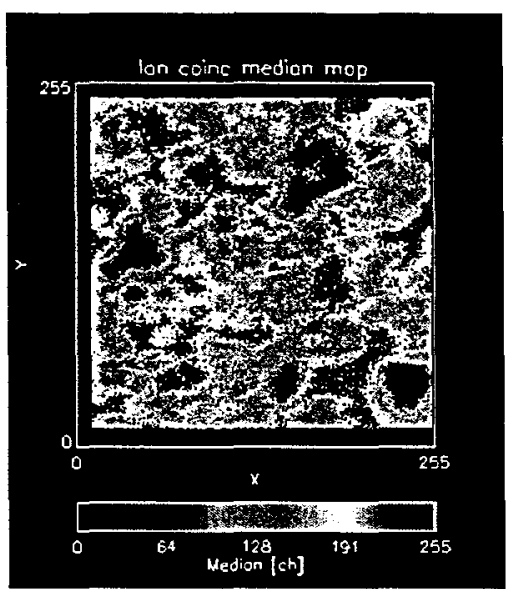

$5 b$

Fig. 5: Ionolumenscence and IBICC median images of a Thiogallate phosphor using $20 \mathrm{MeV} \mathrm{C}$ ions. 5a plots the median of the PMT output which corresponds to the number of photons per ion (e.g. blue represents single photon detection from thin regions while the yellow areas indicate as many as 3 to 4 photons are being collected from thicker regions). 5b shows a medium filtered IBICC image, and here the contrast is reversed: the yellow areas with high IBICC medians are for regions of the thinnest phosphors, and visa versa for the blue low median IBICC regions.

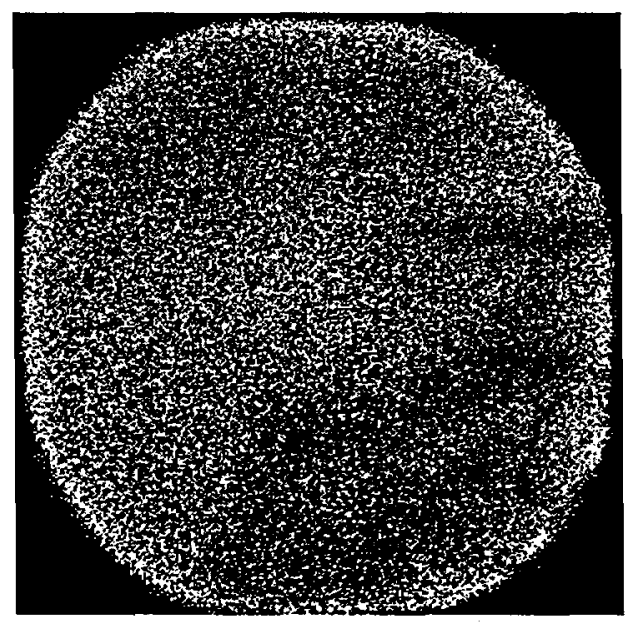

Fig. 6: Image of a scratch on a quartz lens based on electron emission yield contrast. The field of view is $0.45 \mathrm{~mm}$, the magnification was $90 \mathrm{x}$. Charging of the target was suppressed with a pulsed, low energy electron gun. 


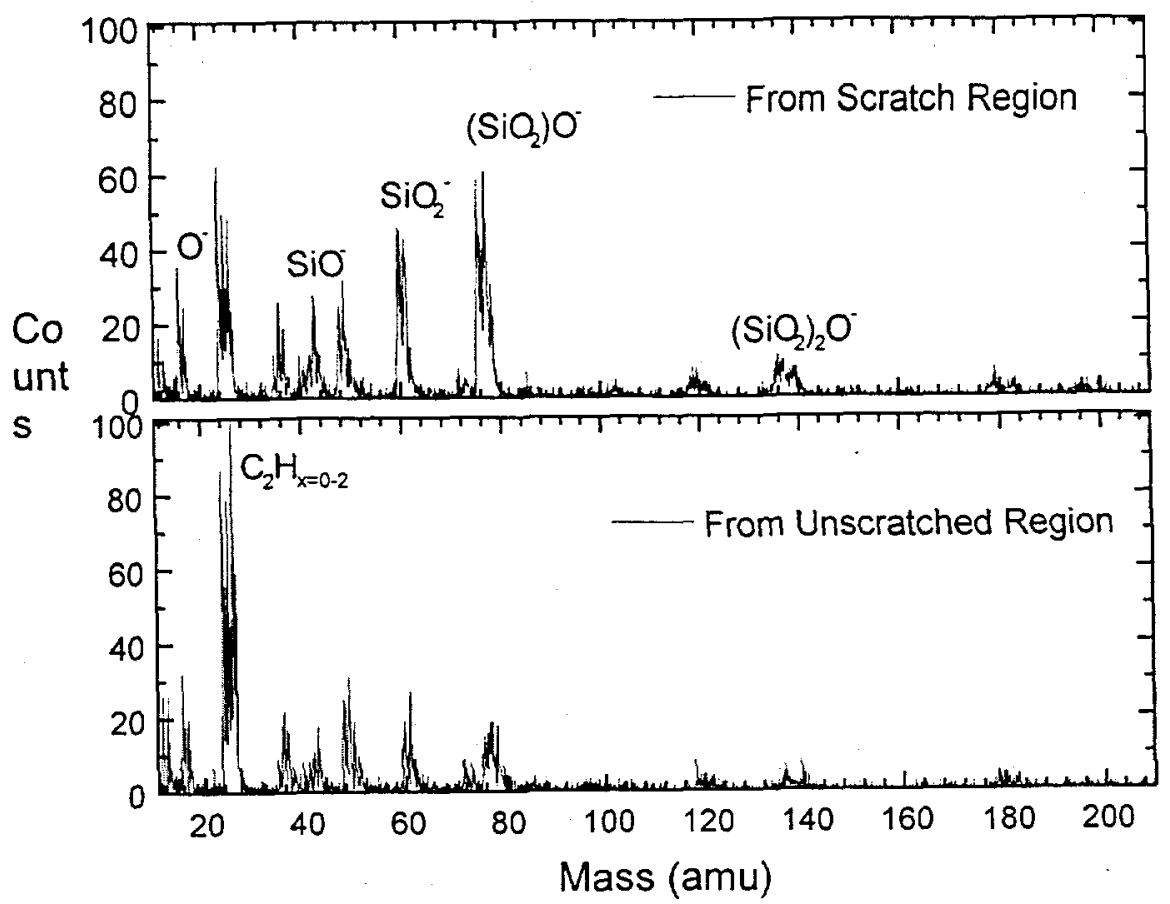

Fig. 7: Negative secondary ion spectra collected from scratched (top) and unscratched (bottom) regions in the image. 